https://doi.org/10.15407/dopovidi2019.12.027

УдК 539.3

\title{
М.М. Діхтярук
}

Хмельницький національний університет

E-mail: mega-dihtyaruk@ukr.net

\section{Плоска контактна задача для пружної смуги з початковими напруженнями, підсиленої нескінченним неоднорідним стрингером}

\author{
Представлено академіком НАН Украйни О.М. Гузем
}

В рамках лінеаризованої теорї пружності розглядається плоска контактна задача про передачу навантаження від нескінченного неоднорідного стрингера до затисненої однією гранню пружної смуги з початковими (залишковими) напруженнями. Дослідження проведені в загальному вигляді для теорії великих початкових деформачій $і$ різних варіантів теорії малих початкових деформачій при довільній структурі пружного потениіалу. Вивчається вплив наявності початкових (залишкових) напружень в смузі на закон розподілу контактних напружень по лінії контакту з нескінченним неоднорідним стрингером. Виходячи 3 припущення про те, що стрингер одночасно навантажується вертикальними і горизонтальними силами, слід зазначити, що стрингер в вертикальному напрямку згинається як звичайна балка, а в горизонтальному напрямку стискується або розтягується, як звичайний стрижень з кінщевою жорсткістю, який знаходиться в одновісному напружено-деформованому стані. Задача математично формулюється як система інтегро-диференціальних рівнянь щодо невідомих контактних напружень. Надалі отримується розв'язуюча система рекурентних систем інтегро-диференціальних рівнянь. Використовуючи перетворення Фур' $\epsilon$, система розв'язується в замкнутому виаляді. В кінцевому результаті вирази для контактних напружень представлені у вигляді інтегралів Фур'є.

Ключові слова: лінеаризована теорія пружності, пружна накладка, початкові (залишкові) напруження, початкові деформачії.

Як відомо, одним із найпоширеніших на практиці способів передачі зовнішніх зусиль $є$ контактна взаємодія. Тому дослідження питань контактної взаємодії твердих тіл, тобто контактні задачі, є досить актуальними. Ця проблема важлива як для фундаментальних розробок в механіці твердого деформівного тіла так і для інших додатків до різних галузей сучасної техніки.

Аналогічна задача у випадку відсутності початкових напружень вивчалася в роботах $[1,2]$. Випадок підсилення попередньо напруженої смуги однорідним нескінченним стрингером розглядався в роботі [4].

(C) М.М. Діхтярук, 2019 
У даній роботі в рамках лінеаризованої теорії пружності [6] досліджується вплив початкових напружень на розподіл контактних напружень в пружній смузі з початковими (залишковими) напруженнями по лінії з'єднання іiї з неоднорідним нескінченним стрингером зі слабкою неоднорідністю.

Будемо вважати, що пружна однорідна смуга з початковими напруженнями товщиною $t$ защемлена гранню $y_{2}=-t$, а на іншій своїй грані підсилена неоднорідним нескінченним стрингером малої товщини $h$.

Нехай, підсилена таким чином смуга піддається дії вертикальних і горизонтальних сил інтенсивності $p_{0}\left(y_{1}\right)$ i $q_{0}\left(y_{1}\right)$

Згідно з прийнятими в [3,4] припущенями, задачу можна сформулювати у вигляді системи

$$
\begin{aligned}
& \frac{d^{2}}{d y_{1}^{2}}\left[D\left(y_{1}\right) \frac{d^{2} u_{2}\left(y_{1}\right)}{d y^{2}}\right]=p\left(y_{1}\right)-p_{0}\left(y_{1}\right), \\
& E_{1}\left(y_{1}\right) h \frac{d u_{1}\left(y_{1}\right)}{d y_{1}}=\int_{-\infty}^{y_{1}}\left[q(\tau)-q_{0}(\tau)\right] d \tau \quad\left(-\infty \prec y_{1} \prec \infty\right),
\end{aligned}
$$

де $D\left(y_{1}\right)=I E_{1}\left(y_{1}\right)$ - жорсткість стрижня на згин.

Нехай матеріал стрижня володіє слабкою неоднорідністю, що змінюється за законом

$$
E_{1}\left(y_{1}\right)=E\left[\left(1+\delta f\left(y_{1}\right)\right] \quad\left(-\infty \prec y_{1} \prec \infty\right),\right.
$$

де $\left.f\left(y_{1}\right)\right]$ - деяка невідома функція; $\delta$ - малий параметр.

Використавши умови контакту, невідомі контактні напруження $p_{0}\left(y_{1}\right), q_{0}\left(y_{1}\right)$ подамо у вигляді ряду за степенями малого параметра:

$$
\begin{aligned}
& p_{0}\left(y_{1}\right)=\sum_{k=0}^{\infty} \delta^{k} p^{(k)}\left(y_{1}\right) \\
& q_{0}\left(y_{1}\right)=\sum_{k=0}^{\infty} \delta^{k} q^{k}\left(y_{1}\right) \quad\left(-\infty \prec y_{1} \prec \infty\right) .
\end{aligned}
$$

3 (1) за допомогою (2), (3), а також формул для знаходження переміщень граничних точок пружної смуги з початковими напруженнями в напрямку осей $0 y_{1}$ i $0 y_{2}$ від одночасної дії нормальних і тангенціальних контактних зусиль для стисливих і нестисливих тіл у випадку пружних потенціалів довільної структури згідно з [1,3] отримаємо

$$
\begin{aligned}
& u_{1}\left(y_{1}\right)=\int_{-\infty}^{\infty} h_{11}\left(\left|y_{1}-\tau\right|\right) p(\tau) d \tau+\int_{-\infty}^{\infty} h_{12}\left(y_{1}-\tau\right) q(\tau) d \tau, \\
& u_{2}\left(y_{1}\right)=\int_{-\infty}^{\infty} h_{21}\left(y_{1}-\tau\right) p(\tau) d \tau+\int_{-\infty}^{\infty} h_{22}\left(\left|y_{1}-\tau\right|\right) q(\tau) d \tau .
\end{aligned}
$$


Тут $h_{i j}\left(y_{1}\right)$ - функції впливу, вирази для яких задаються формулами у випадку дії вертикальної зосередженої сили і у випадку горизонтальної зосередженої сили [4]. Ядра $H_{i j}(\alpha)$ i $\tilde{H}_{i j}(\alpha)$ для рівних і нерівних коренів визначального рівняння $[1,5]$ також визначаються за відомими формулами [4]. Відзначимо, що в подальших дослідженнях ми будемо користуватися виразами для переміщень, в яких в залежності від значень ядер $H_{i j}(\alpha)$ і $\tilde{H}_{i j}(\alpha)$ можна одержати розв'язки для стисливих і нестисливих тіл у випадку рівних і нерівних коренів визначального рівняння $[1,5]$.

В результаті отримаємо розв'язуючу систему рекурентних систем інтегро-диференційних рівнянь

$$
\begin{aligned}
& D_{0} \frac{d^{4} u_{2}^{(0)}\left(y_{1}\right)}{d\left(y_{1}\right)^{4}}=p^{(0)}\left(y_{1}\right)-p_{0}\left(y_{1}\right) \quad\left(-\infty \prec y_{1} \prec \infty\right), \\
& E_{0} h \frac{d^{2} u_{1}^{(0)}\left(y_{1}\right)}{d\left(y_{1}\right)^{2}}=q^{(0)}\left(y_{1}\right)-q_{0}\left(y_{1}\right), \\
& D_{0} \frac{d^{4} u_{2}^{(k)}\left(y_{1}\right)}{d\left(y_{1}\right)^{4}}=p^{(k)}\left(y_{1}\right)-f_{1}^{(k-1)}\left(y_{1}\right) \quad(k=1,2, \ldots), \\
& E_{0} h \frac{d^{2} u_{1}^{(k)}\left(y_{1}\right)}{d\left(y_{1}\right)^{2}}=q^{(k)}\left(y_{1}\right)-f_{2}^{(k-1)}\left(y_{1}\right),
\end{aligned}
$$

де $D_{0}=E_{0} I$,

$$
\begin{aligned}
& u_{1}\left(y_{1}\right)=\int_{-\infty}^{\infty} h_{11}\left(\left|y_{1}-\tau\right|\right) p^{(k)}(\tau) d \tau+\int_{-\infty}^{\infty} h_{12}\left(y_{1}-\tau\right) q^{(k)}(\tau) d \tau \\
& \left(-\infty \prec y_{1} \prec \infty, k=0,1, \ldots\right) \\
& u_{2}\left(y_{1}\right)=\int_{-\infty}^{\infty} h_{21}\left(y_{1}-\tau\right) p^{(k)}(\tau) d \tau+\int_{-\infty}^{\infty} h_{22}\left(\left|y_{1}-\tau\right|\right) q^{(k)}(\tau) d \tau, \\
& f_{1}^{(k-1)}\left(y_{1}\right)=D_{0} \frac{d^{2}}{d\left(y_{1}\right)^{2}}\left[f\left(y_{1}\right) \frac{d^{2} u_{2}{ }^{(k-1)}\left(y_{1}\right)}{d\left(y_{1}\right)}\right] \quad(k=1,2, \ldots) \\
& f_{2}{ }^{(k-1)}\left(y_{1}\right)=E_{0} h \frac{d}{d\left(y_{1}\right)}\left[f\left(y_{1}\right) \frac{d u_{1}^{(k-1)}\left(y_{1}\right)}{d\left(y_{1}\right)}\right] .
\end{aligned}
$$

Система (5) описує контактну задачу для однорідного нескінченного стрингера [4], кожна наступна система з (6) відрізняється від попередньої тільки зовнішнім навантаженням. Отже, розв'язування задачі для попередньо напруженої смуги, підсиленої неоднорід- 
ним нескінченним стрингером, зводиться до розв’язування кількох однорідних задач, які відрізняються між собою тільки прикладеними зовнішніми навантаженнями.

Нульове наближення розв'язку неоднорідної задачі, тобто розв'язок системи (5) за допомогою перетворення Фур'є будується аналогічно описаному в [2] і набуває вигляду

$$
\begin{aligned}
& p\left(y_{1}\right)=\frac{\mu}{2 \pi} \int_{-\infty}^{\infty}\left[\alpha^{2} H_{21}^{*}(\alpha) \tilde{q}_{0}(\alpha)+H_{22}^{*}(\alpha) \tilde{p}_{0}(\alpha)\right] H^{-1}(\alpha) e^{-i \alpha y_{1}} d \alpha \quad\left(-\infty \prec y_{1} \prec \infty\right) \\
& q\left(y_{1}\right)=\frac{\mu}{2 \pi} \int_{-\infty}^{\infty}\left[H_{11}^{*}(\alpha) \tilde{q}_{0}(\alpha)-i H_{12}^{*}(\alpha) \tilde{p}_{0}(\alpha)\right] H^{-1}(\alpha) e^{-i \alpha y_{1}} d \alpha
\end{aligned}
$$

Тут величини $H_{i j}^{*}(i, j=1,2)$ виражаються через відомі функції $H_{i j}^{*}(i, j=1,2)$, які визначаються згідно з формулами для рівних і нерівних коренів визначального рівняння $[1,4]$ у випадку конкретної структури пружних потенціалів.

Решта наближених розв'язків, які є результатом впливу неоднорідності матеріалу стрингера, будуються аналогічно.

Так $k$-те наближення має вигляд

$$
p^{(k)}\left(y_{1}\right)=\frac{1}{2 \pi} \int_{-\infty}^{\infty} P^{(k)}(s) e^{-i s y_{1}} d s, q^{(k)}\left(y_{1}\right)=\frac{1}{2 \pi} \int_{-\infty}^{\infty} Q^{(k)}(s) e^{-i s y_{1}} d s \quad(k=1,2, \ldots),
$$

де

$$
\begin{aligned}
& P^{(k)}(s)=D s^{2}\left\{\bar{f}_{1}^{(k-1)}(s)\left[E_{0} h s^{2} H_{22}(s)+1\right]-E_{0} h s^{3} \bar{f}_{2}^{(k-1)}(s) H_{12}(s)\right\} / L(s) \quad(k=1,2, \ldots), \\
& Q^{(k)}(s)=-I E_{0} h s\left\{\bar{f}_{2}^{(k-1)}(s)\left[D_{0} h s^{4} H_{11}(s)+1\right]+D_{0} h s^{3} \bar{f}_{1}^{(k-1)}(s) H_{12}(s)\right\} / L(s)
\end{aligned}
$$

і є трансформантами Фур’є контактних напружень.

Тут

$$
\begin{aligned}
& L(s)=\left[D_{0} s^{4} H_{11}(s)-1\right]\left[E_{0} h s^{2} H_{22}(s)+1\right]+D_{0} E_{0} s^{4} h H_{12}^{2}(s), \\
& \bar{f}_{j}^{(k-1)}(s)=F\left[f_{j}^{(k-1)}\left(y_{1}\right)\right] \quad(j=1,2), \quad(k=1,2, \ldots),
\end{aligned}
$$

а $H_{i j}(s)$ і $H_{i j}\left(y_{1}\right) \quad(i, j=1,2)$ задаються згідно з [4] відомими формулами.

Таким чином, цим методом можна розв’язувати велику кількість контактних задач для пружних тіл з початковими напруженнями, підсиленими нескінченним неоднорідним стрингером зі слабкою неоднорідністю. В результаті будуть одержані рекурентні системи, розв’язки яких будуються за степенями малого параметра.

\section{ЦИТОВАНА ЛІТЕРАТУРА}

1. Арутюнян И.Х., Мхитарян С.М. Некоторые контактные задачи для полуплоскости с частично скрепленными упругими накладками. Изв. АН Арм. ССР. Механика. 1972. 25, № 2. С. 15-37.

2. Саркисян В.С. Контактные задачи для полуплоскостей и полос с упругими накладками. Ереван: Изд-во Ереван. ун-та, 1983. 260 с. 
3. Guz A.N., Babich S.Y., Rudnitskii V.B. Contact problems for elastic bodies with initial stresses: Focus on Ukrainian research. Int. Appl. Mech. Rew. 1998. 51, № 5. P. 343-371.

4. Рудницкий В.Б., Дихтярук Н.Н. Упругая полоса с начальными напряжениями, усиленная упругими накладками. Прикл. механика. 2002. 38, № 11. С. 81-88.

5. Гузь А.Н., Бабич С.Ю., Рудницький В.Б. Контактна взаємодія тіл з початковими напруженнями. Київ: Вища шк., 1995. 305 с.

6. Гузь А.Н. Механика хрупкого разрушения материалов с начальными напряжениями. Киев: Наук. думка, 1983. 240c.

7. Штаерман И.Я. Контактные задачи теории упругости. Москва: ГИТТЛ. 1949. 270 с.

Надійшло до редакції 11.07.2019

\section{REFERENCES}

1. Arutyunyan, N. Kh. \& Mhitaryan, S. M. (1968). Some contact problems for a half-plane with partially fastened elastic overlays. Izv. AS Arm. SSR. Mechanics, 32, No. 4, pp. 632-646.

2. Sarkisyan, V. S. (1983). Contact problems for the half-planes and strips with elastic straps. Yerevan: Yerevan Univ. Press (in Russian).

3. Guz, A. N., Babich, S. Y. \& Rudnitskii, V. B. (1998). Contact problems for elastic bodies with initial stresses: Focus on Ukrainian research. Int. Appl. Mech. Rew., 51, No. 5, pp. 343-371.

4. Rudnitskii, V. B. \& Dihtyaruk, N. N. (2002). Elastic strip with initial stresses, reinforced by elastic linings. Prikl. Mekh. 38, No. 11, pp. 81-88 (in Russian).

5. Guz, A. N., Babich, S. Y. \& Rudnitskii, V. B. (1995). Contact interaction of bodies with initial stresses. Kyiv: Vysha Schcola (in Ukrainian).

6. Guz, A. N. (1983). Mechanics of brittle fracture of materials with initial stresses. Kiev: Naukova Dumka.

7. Shtaerman, I. Ya. (1949). Contact problems of the theory of elasticity. Moscow: GITTL (in Russian).

Received 11.07.2019

\section{Н.Н. Дихтярук}

Хмельницкий национальный университет

E-mail: mega-dihtyaruk@ukr.net

ПЛОСКАЯ КОНТАКТНАЯ ЗАДАЧА ДЛЯ УПРУГОЙ ПОЛОСЫ

С НАЧАЛЬНЫМИ НАПРЯЖЕНИЯМИ, УСИЛЕННОЙ

БЕСКОНЕЧНЫМ НЕОДНОРОДНЫМ СТРИНГЕРОМ

В рамках линеаризированной теории упругости рассматривается плоская контактная задача о передаче нагрузки от бесконечного неоднородного стрингера к защемленной одной гранью упругой полосы с начальными (остаточными) напряжениями. Исследования проведены в общем виде для теории больших начальных деформаций и разных вариантов теории малых начальных деформаций при произвольной структуре упругого потенциала. Изучается влияние наличия начальных (остаточных) напряжений в полосе на закон распределения контактных напряжений по линии контакта с бесконечным неоднородным стрингером. Исходя из предположения о том, что стрингер одновременно нагружается вертикальными и горизонтальными силами, следует отметить, что стрингер в вертикальном направлении изгибается как обычная балка, а в горизонтальном направлении сжимается или растягивается, как обычный стержень с конечной жесткостью, который находится в одноосном напряженно-деформированном состоянии. Задача математически формулируется как система интегро-дифференциальных уравнений относительно неизвестных контактных напряжений. В дальнейшем получается разрешающая система рекуррентных систем интегро- дифференциальных уравнений. Используя преобразования Фурье, система решается в замкнутом виде. В конечном итоге выражения для контактных напряжений представлены в виде интегралов Фурье.

Ключевые слова: линеаризованная теория упругости, упругая накладка, начальные (остаточные) напряжения, начальнье деформащии. 


\section{N.N. Dikhtyaruk}

Khmel'nyts'k National University

E-mail: mega-dihtyaruk@ukr.net

\section{THE PLANE CONTACT PROBLEM FOR}

AN INITIALLY STRESSED ELASTIC STRIP REINFORCED BY AN INFINITE INHOMOGENEOUS STRINGER

In the frame of linearized elasticity theory, the plane contact problem of load transmission from an infinite inhomogeneous stringer to an elastic strip which is jammed at one edge and undergoes the action of initial (residual) stresses is considered. The study is carried out in the general form within the theory of high initial strains, as well as within various versions of the theory of low initial strains, at any structure of the elastic potential. The effect of the presence of initial (residual) stresses in the strip on the law of distribution of stresses on the line of contact with the infinite inhomogeneous stringer is studied. If the stringer is simultaneously loaded by vertical and horizontal forces, it is bent in the vertical direction as an ordinary beam and is compressed or stretched as an ordinary rod with finite stiffness, being in the uniaxial stress-strain state. The problem is posed mathematically as a system of integro-differential equations for the unknown contact stresses. Then the resolving system of recurrence systems of integro-differential equations is constructed and solved in the closed form with the use of the Fourier transformation. The contact stresses are given in the form of Fourier integrals.

Keyzords: linearized elasticity theory, elastic strap, initial (residual) stresses, initial deformations. 